\title{
Local detection of three-dimensional inclusions in electrical impedance tomography
}

\author{
T Ide, H Isozaki, S Nakata and S Siltanen
}

\begin{abstract}
Assume given a three-dimensional bounded domain with an unknown conductivity distribution inside. Further, suppose that the conductivity consists of a known background and unknown anomalous regions (inclusions) where conductivity values are unknown and different from the background. A method is introduced in [Ide et al., Comm Pure Appl Math 60, 2007] for locating inclusions approximately from noisy localized voltage-to-current measurements performed at the boundary of the body. The method is based on the use of complex geometrical optics solutions and hyperbolic geometry; numerical testing is presented in the aforementioned paper for the two-dimensional case. This work reports the results of computational implementation of the method in dimension three, where both the simulation of data and the computerized inversion algorithm are more complicated than in dimension two. Three new regularizing steps are added to the algorithm, resulting in significantly better robustness against noise. Numerical experiments are reported, suggesting that approximate location of the inclusions can be reliably recovered from data with realistic level of measurement noise. Potential applications of the results include early diagnosis of breast cancer, underground contaminant detection and nondestructive testing.
\end{abstract}

Revised manuscript, November 12, 2009

\section{Introduction}

Assume given a three-dimensional bounded domain with a strictly positive conductivity distribution inside. Further, suppose that the conductivity consists of a known background and possible anomalous regions (inclusions) where conductivity values are unknown and different from the background. A method is introduced in [43] for locating inclusions approximately from noisy local voltage-to-current measurements performed at the boundary of the body. That method is based on the use of complex geometrical optics solutions and hyperbolic geometry; one essentially probes the unknown body with balls and decides, using boundary measurements localized inside the ball, whether the ball intersects an inclusion or not. Numerical testing is presented in [43] for the two-dimensional case. In this paper we explain how to implement the method in a three-dimensional setting and demonstrate it on simulated noisy data. Further, three new regularizing steps are added to the algorithm described in [43], resulting in significantly better robustness against measurement noise.

Our results can be seen as a new imaging method for three-dimensional electrical impedance tomography (EIT), applications of which include early diagnosis of breast cancer, underground contaminant detection and nondestructive testing. See [28] for a review of EIT. 
Let $\Omega$ be a bounded open set with smooth boundary in $\mathbb{R}^{3}$ and consider the following boundary value problem:

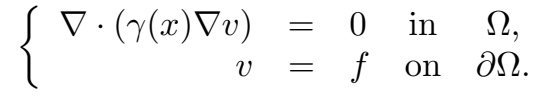

We assume that $\gamma \in L^{\infty}(\Omega)$ and that $\gamma(x) \geq c_{0}>0$ almost everywhere in $\Omega$. Define the Dirichlet-to-Neumann (DN) map $\Lambda_{\gamma}: H^{1 / 2}(\partial \Omega) \rightarrow H^{-1 / 2}(\partial \Omega)$ by

$$
\Lambda_{\gamma}:\left.f \rightarrow \gamma(x)\left(\frac{\partial v}{\partial n}\right)\right|_{\partial \Omega},
$$

where $v$ is the solution to (1.1) and $n$ is the outer unit normal to $\partial \Omega$. Here $\gamma$ represents the electric conductivity inside a physical body, $v$ is the voltage potential, and the linear operator $\Lambda_{\gamma}$ models static voltage-to-current measurements at the boundary.

The inverse conductivity problem of Calderón [25] has two parts: uniqueness and reconstruction. The uniqueness question is to find out whether $\gamma$ is uniquely determined by $\Lambda_{\gamma}$, and the reconstruction task is to calculate $\gamma(x)$ given the knowledge of $\Lambda_{\gamma}$. Since the reconstruction problem is severely ill-posed, in practical EIT it is important to design reconstruction algorithms that are robust against measurement noise. Moreover, it is often enough to recover only some features of the conductivity, such as location of inclusions, instead of the point values $\gamma(x)$ for all $x \in \Omega$.

We consider detection of inclusions in a three-dimensional body $\Omega$ from local boundary measurements done on a fixed subset $\Gamma \subset \partial \Omega$. Let a background conductivity $\gamma_{0}(x) \in C^{\infty}(\bar{\Omega})$ be given. Assume that $\Omega_{1}$ is an open set with piecewise smooth boundary $\partial \Omega_{1}$ and that $\bar{\Omega}_{1} \subset \Omega$. We consider conductivities of the form

$$
\gamma(x)= \begin{cases}\gamma_{1}(x), & x \in \Omega_{1}, \\ \gamma_{0}(x), & x \in \Omega_{0}:=\Omega \backslash \bar{\Omega}_{1},\end{cases}
$$

with $\gamma_{1}(x) \in L^{\infty}(\Omega)$ such that $\gamma(x) \geq c_{0}>0$ almost everywhere in $\Omega$. Further, we assume that $\gamma_{1}(x)-\gamma_{0}(x)$ has a constant sign on $\Omega_{1}$, and for any $p \in \Omega_{1}$, there exist constants $C>0$ and $\epsilon>0$ such that $C^{-1}<\left|\gamma_{1}(x)-\gamma_{0}(x)\right|<C$ if $|x-p|<\epsilon$.

Define the associated Dirichlet-to-Neumann maps by

$$
\Lambda_{0}:\left.f \rightarrow \gamma_{0}\left(\frac{\partial u}{\partial n}\right)\right|_{\partial \Omega}, \quad \Lambda:\left.f \rightarrow \gamma\left(\frac{\partial v}{\partial n}\right)\right|_{\partial \Omega},
$$

where $v$ is the solution to (1.1) and $u$ solves equation (1.1) with $\gamma$ replaced by $\gamma_{0}$. Now the problem is to find information about the inclusion set $\Omega_{1}$ from the knowledge of $\Lambda_{0} f$ and $\Lambda f+c \eta$, where $f$ may range over a collection of functions supported on $\Gamma \subset \partial \Omega$. Here $\eta$ is random measurement error modelled by white noise with unit standard deviation, and $c \geq 0$ is the noise amplitude.

Now Theorem 1.1 of [43] outlines how to detect inclusions from infinite presicion data (i.e. $c=0$ ) by probing with balls:

(i) Place the body $\Omega$ in the upper half space $\mathbb{R}_{+}^{3}$ so that $\partial \Omega$ has a positive distance from $\left\{x \in \mathbb{R}^{3} \mid x_{d}=0\right\}$.

(ii) Draw a ball $B(0, R)$ such that $(\overline{B(0, R)} \cap \partial \Omega) \subset \Gamma$.

(iii) For large $\tau>0$, compute $I(\tau)=\left\langle\left(\Lambda-\Lambda_{0}\right) f_{\tau}, f_{\tau}\right\rangle_{\Gamma}$.

(iv) If $I(\tau) \rightarrow 0$ as $\tau \rightarrow \infty$, we infer that $B(0, R)$ does not intersect the inclusion. 
(v) If $I(\tau) \rightarrow \infty$ as $\tau \rightarrow \infty$, we infer that $B(0, R) \cap \Omega_{1} \neq \emptyset$ and that $\gamma_{1}(x)>\gamma_{0}(x)$ inside the inclusion $\Omega_{1}$. If $I(\tau) \rightarrow-\infty$ as $\tau \rightarrow \infty$, we infer that $B(0, R) \cap \Omega_{1} \neq \emptyset$ and that $\gamma_{1}(x)<\gamma_{0}(x)$ inside the inclusion.

The essential part of the above method is the choice of Dirichlet data $f_{\tau}$. In the constant background case $\gamma_{0} \equiv 1$ the functions $f_{\tau}$ take a particularly simple form, see Section 2.2 below. For more general background conductivities we refer to [43] for the construction of appropriate Dirichlet data.

In practical situations when $c>0$ it is not possible to compute the limit $\tau \rightarrow \infty$ since the measurement noise will be multiplied by a factor that depends exponentially on $\tau$, and the Dirichlet data corresponding to large $\tau$ values is very oscillatory and cannot be approximated by practical electrode measurements. Therefore, following [43], the steps (iii), (iv) and (v) are replaced by

(iii) ${ }^{\prime}$ Take $0<\tau_{1}<\tau_{2}$ and compute the corresponding inner products

$$
I_{1}:=\left\langle\left(\Lambda-\Lambda_{0}\right) f_{\tau_{1}}, f_{\tau_{1}}\right\rangle_{\Gamma} \quad \text { and } \quad I_{2}:=\left\langle\left(\Lambda-\Lambda_{0}\right) f_{\tau_{2}}, f_{\tau_{2}}\right\rangle_{\Gamma} .
$$

(iv) ${ }^{\prime}$ Use the noise level $c>0$ to choose a threshold $\varepsilon_{\mathrm{T}}>0$ as explained in Section 3.2.3 below. If $\left|I_{1}\right|<\varepsilon_{\mathrm{T}}$ or $\left|I_{2}\right|<\varepsilon_{\mathrm{T}}$, then we infer that $B(0, R)$ does not intersect the inclusion. Otherwise, continue to Step $(\mathrm{v})^{\prime}$.

$(\mathrm{v})^{\prime}$ If $I_{1} \geq I_{2}>0$ or $I_{1} \leq I_{2}<0$, we infer that $B(0, R)$ does not intersect the inclusion.

(vi) $^{\prime}$ If $I_{2}>I_{1}>0$, then we infer that $B(0, R) \cap \Omega_{1} \neq \emptyset$ and that $\gamma_{1}(x)>\gamma_{0}(x)$ inside the inclusion $\Omega_{1}$. If $I_{2}<I_{1}<0$, then we infer that $B(0, R) \cap \Omega_{1} \neq \emptyset$ and that $\gamma_{1}(x)<\gamma_{0}(x)$ inside the inclusion.

Of course, steps (iii) $)^{\prime}$ (iv) $)^{\prime}$ and $(\mathrm{v})^{\prime}$ give only approximate information whereas steps (iii), (iv) and (v) hold exactly. However, the necessity of using the modified steps is a consequence of the inherent ill-posedness of the inverse conductivity problem. Note that our algorithm does not involve the solution of any direct problem, so it is computationally effective.

We introduce the following additional techniques to improve the robustness of inclusion detection:

(a) rotation of Dirichlet boundary conditions,

(b) restriction of probing depth, and

(c) stabilization of distance estimation process.

The process (a) enables us to reduce the effect of asymmetric distribution of Dirichlet data to the accuracy of the distance estimation. The restriction of the probing depth in (b) is employed in order to avoid inaccurate approximation of the distance in the case that the distance from a center point to the inclusion is large. The third one, (c), is to stabilize the inclusion detection algorithm in the case that measurement noise results in large error in the inner product.

Let us review the relevant literature on the inverse conductivity problem. We restrict our review to results in dimension three.

The first uniqueness result was given by Kohn and Vogelius [59] for piecewise analytic conductivities. Uniqueness for infinitely smooth conductivities was proved by Sylvester and Uhlmann in [75], and the smoothness requirement was later relaxed in $[1,21,27,39,70,72,74]$. The above works assume measuring on the whole boundary; 
uniqueness proofs for local data are given in [24, 54, 56, 57, 71]. Curiously, [59] is still the only result establishing global uniqueness in dimension three for a class containing discontinuous conductivities.

Reconstruction of the full conductivity in 3D was discussed by Calderón for the linearized problem in [25]; a numerical implementation in dimension three was done in [20]. First theoretical reconstructions were given, independently, in [73] and [70]. Numerical reconstruction method based on [70] was discussed in [30] and implemented in [18] and [20]. Practical reconstruction methods based on regularization and aiming at full reconstruction in $3 \mathrm{D}$ are reported in $[19,38,64,65,66,67,77,78,79,80]$.

The study of detecting inclusions was initialized in $[33,34]$. Important uniqueness results include $[2,4,5,35,53,59]$. Local measurements are known to yield information about discontinuities in conductivity in the case of a bounded domain [44, 46, 49], an infinite slab [47], and multilayered medium [48]. In the case of polyhedra, uniqueness was established in [17, 57]. Characterization of inclusions using variants of the factorization method has been described in [22, 41, 42, 62], and practical recovery of inclusions is reported in [40,37]. A numerical inclusion detection method is proposed in [55]. Recovering medically relevant information from local boundary measurements is discussed in $[68,69]$. There is an extensive body of work aiming at the estimation of the size of inclusion: $[7,8,9,60,6,3,45,63,31]$. For methods dedicated to detecting small inhomogeneities, see $[11,13,15,26,10,12,14,16,61]$.

The use of complex geometrical optics solutions for numerical detection of inclusions was initiated (simultaneously and independently) in [23, 50] by probing with half-planes using the enclosure method of Ikehata [46]. That approach was later generalized to probing with cones in [51], with balls in [43], and with more general shapes in [76]. The present work is the first three-dimensional implementation of a detection method based on complex geometrical optics.

This paper is organized as follows. In Section 2 we test the method in the case of layered medium, where the ground truth can be computed analytically for comparison. Section 3 is devoted to a numerical study of detecting three-dimensional inclusions from localized noisy EIT data. In particular, Section 3.2 provides a discussion of the three steps we take to add robustness of the method against noise. Finally, we conclude our results in Section 4.

\section{Layered medium}

Let us consider a simple setting of material with one interface between two layers of different conductivities. This case can be used to test our computer algorithms since some functions that appear in this case can be computed analytically.

\subsection{Geometry of the problem}

We take $\Omega$ to be the square

$$
\Omega=\left\{\left(x_{1}, x_{2}, x_{3}\right):-1 \leq x_{1} \leq 1, \quad-1 \leq x_{2} \leq 1, \quad 0 \leq x_{3} \leq 2\right\} \subset \mathbb{R}^{3},
$$

and denote the bottom boundary of $\Omega$ by $\Gamma$ :

$$
\Gamma=\left\{\left(\left(x_{1}, x_{2}, x_{3}\right):-1 \leq x_{1} \leq 1,-1 \leq x_{2} \leq 1, x_{3}=0\right)\right\}
$$


The voltage-to-current measurements are performed on a localized area in $\Gamma$. The inclusion we assume in this problem is the layered area given by

$$
\Omega_{1}=\left\{\left(x_{1}, x_{2}, x_{3}\right):-1 \leq x_{1} \leq 1, \quad-1 \leq x_{2} \leq 1, \quad h \leq x_{3} \leq 2\right\} \subset \mathbb{R}^{3},
$$

where $h$ is the height of the interface from the bottom as shown in Fig. 1. Assume the background conductivity $\gamma_{0}=1.0$, the conductivity of inclusion $\gamma_{1}=4.0$ and the center point $x_{0}=(-0.2,0,0)$. The goal of this test problem is to estimate the distance from $x_{0}$ to the inclusion using the localized voltage-to-current measurements.

\subsection{Dirichlet data}

An approximation of the voltage distribution used for determining inclusions, as suggested in [43], is given by

where

$$
u_{\tau}(x) \simeq \sqrt{\frac{y_{3}}{x_{3}}} e^{-\tau y_{1}+i \tau y_{3}},
$$

$$
y_{1}=\frac{x_{1}^{2}+x_{2}^{2}+x_{3}^{2}-R^{2}}{\left(x_{1}+R\right)^{2}+x_{2}^{2}+x_{3}^{2}}, \quad y_{3}=\frac{2 x_{3} R}{\left(x_{1}+R\right)^{2}+x_{2}^{2}+x_{3}^{2}} .
$$

We use $f_{\tau}$ defined above as the Dirichlet data imposed on the localized boundary $B\left(x_{0}, R\right) \cap \partial \Omega$ with truncation so that the support of the voltage is restricted inside the ball, i.e.

$$
f_{\tau}=\left\{\begin{array}{cl}
u_{\tau}, & x \in B\left(x_{0}, R\right) \cap \partial \Omega, \\
0, & \text { otherwise. }
\end{array}\right.
$$

Examples of the Dirichlet data on $\Gamma$ corresponding to different radii with $\tau=2$ and 2.5 are shown in Fig. 2. The figure illustrates the real part of $f_{\tau}$. The imaginary part also behaves in a way similar to the corresponding real part, i.e. the voltage vanishes to zero as a point on the localized boundary approaches to its edge. Although the increase of $\tau$ results in higher fluctuation in the boundary voltage as seen in this figure and as supposed from (2.1), the modified version of our algorithm, (iii)', (iv) ${ }^{\prime}$ and $(\mathrm{v})^{\prime}$, avoids the situation and allows us to run the procedure even if $\tau$ 's are not large enough to see the limit value of the inner product in (iv). The asymmetric and nonuniform distribution of the voltage, as shown also in Fig. 2, arises from the coordinate transform in (2.2) and results in asymmetric performance of the inclusion detection algorithm, i.e. the accuracy of the inclusion detection depends on the coordinate system. In section 3 we provide an idea to solve this drawback.

\subsection{Dirichlet-Neumann map for homogeneous conductivity}

Let us consider the following Dirichlet problem with homogeneous conductivity:

$$
\begin{cases}\Delta u=0 & \text { in } \Omega, \\ u=\varphi_{m n}\left(x_{1}, x_{2}\right) & \text { on } \Gamma, \\ u=0 & \text { on } \partial \Omega \backslash \Gamma,\end{cases}
$$

where $\varphi_{m n}\left(x_{1}, x_{2}\right)=\sin \left\{\pi m\left(x_{1}+1\right) / 2\right\} \sin \left\{\pi n\left(x_{2}+1\right) / 2\right\}$. The functions $\varphi_{m n}$ are eigenfunctions of the DN map, i.e. $\Lambda_{0} \varphi_{m n}=\lambda_{m n}^{(0)} \varphi_{m n}$, where $\lambda_{m n}^{(0)}$ are the eigenvalues correspoinding to $\varphi_{m n}$ given by

$$
\lambda_{m n}^{(0)}=\frac{\sqrt{m^{2}+n^{2}} \pi\left(1+e^{-2 \sqrt{m^{2}+n^{2}} \pi}\right)}{2\left(1-e^{-2 \sqrt{m^{2}+n^{2}} \pi}\right)} .
$$




\subsection{Dirichlet-Neumann map for layered medium}

Let us consider the following Dirichlet problem with layered conductivity:

$$
\begin{cases}\nabla \cdot(\gamma \nabla u)=0 & \text { in } \Omega, \\ u=\varphi_{m n}\left(x_{1}, x_{2}\right) & \text { on } \Gamma, \\ u=0 & \text { on } \partial \Omega \backslash \Gamma,\end{cases}
$$

where $\varphi_{m n}\left(x_{1}, x_{2}\right)=\sin \left\{\pi m\left(x_{1}+1\right) / 2\right\} \sin \left\{\pi n\left(x_{2}+1\right) / 2\right\}$ and $\gamma$ is the layered conductivity with an interface at $x_{3}=h$ as defined in subsection 2.1. The DN map of $\varphi_{m n}$ can be written as $\Lambda \varphi_{m n}=\lambda_{m n} \varphi_{m n}$, where $\lambda_{m n}$ are the eigenvalues of $\Gamma$ given by

$$
\lambda_{m n}=\alpha \frac{(\sigma-1)\left(e^{2 \alpha(h-2)}+e^{-2 \alpha h}\right)+(\sigma+1)\left(e^{-4 \alpha}+1\right)}{(\sigma-1)\left(e^{2 \alpha(h-2)}-e^{-2 \alpha h}\right)-(\sigma+1)\left(e^{-4 \alpha}-1\right)}, \quad \alpha=\frac{\sqrt{m^{2}+n^{2}}}{2} \pi .
$$

\subsection{Layered medium: numerical studies}

This numerical test aims at evaluating the performance of the algorithm in the layered medium case. The inner product in (iii)' can be calculated, using the eigenvalues and eigenvectors shown in the previous subsections, as

$$
\left\langle\left(\Lambda-\Lambda_{0}\right) f_{\tau}, f_{\tau}\right\rangle_{\Gamma}=\sum_{m, n}\left|a_{m n}\right|^{2}\left(\lambda_{m n}-\lambda_{m n}^{(0)}\right),
$$

where $a_{m n}$ are the coefficients of the sine series expansion:

$$
f_{\tau}\left(x_{1}, x_{2}\right)=\sum_{m, n} a_{m n} \varphi_{m n}\left(x_{1}, x_{2}\right) .
$$

We fix the height $h$ and compute the inner product (2.3) for varying radius $R$ with two different parameters, $\tau_{1}$ and $\tau_{2}$. The inner product for a fixed radius approximately determines whether the ball intersects with the intersection or not using the criterion in (iv)'. The property in (iv) ${ }^{\prime}$ leads to an approximate distance between $x_{0}$ and $\Omega_{1}$ by taking the maximum radius that satisfies $I_{1} \geq I_{2}$, i.e. the approximate distance is the intersection of two graphs of the inner product drawn as functions of $R$ for two different $\tau$ 's. Fig. 3 illustrates the graphs of the inner products using two different parameters, $\tau_{1}=2.0$ and $\tau_{2}=2.5$. The left columns illustrate the geometry of the problem domain and the inclusion, center columns are the plots of $I_{1}$ and $I_{2}$ computed using the formula (2.3). In order to investigate the effect of noise in measurements, we added white noise to Neumann data by replacing $\Lambda f_{\tau}$ to $\Lambda f_{\tau}+c \eta$, where $\eta$ is the white noise of which standard deviation is 1 and $c$ is noise amplitude satisfying

$$
c=\|\eta\|_{\infty} /\left\|\Lambda f_{\tau}\right\|_{\infty} .
$$

The images in the right columns of Fig. 3 are the plots of $I_{1}$ and $I_{2}$ computed with noise, where noise amplitude $c=1.0 \times 10^{-4}$. Such noise amplitude is attainable by practical measurement devices; for example a signal-to-noise ratio of $95.5 \mathrm{~dB}$ is reported in [29], corresponding to $1.7 \times 10^{-5}$ in our notation.

\section{Homogeneous background with inclusions}

In this section the performance of the algorithm to detect inclusions is examined with arbitrarily located inclusions. The goal is to partially recover the shape of the inclusion using approximate distances from multiple center points to the inclusion. 


\subsection{Geometry of the problem}

Let us consider the same domain $\Omega$ as in section 2 and inclusions arbitrarily located in $\Omega$, where the conductivities of the background and the inclusion are 1 and 4 , respectively. We assume multiple probing balls centered below the bottom boundary at $x_{3}=-0.2$ as shown in the left column of Fig. 5, where the centers are the points given as $10 \times 10$ uniformly located grid points on the square $\left(x_{1}, x_{2}\right) \in[-0.8,0.8] \times[-0.8,0.8]$. Let us denote the center points by $x_{0}^{(j)}(j=1, \ldots, J)$. The radius of each probing ball is chosen so that the intersection of the ball and the boundary of the problem domain is inside the bottom boundary, i.e. $0.2<R \leq R_{\max }\left(x_{0}^{(j)}\right)$, where $R_{\max }\left(x_{0}^{(j)}\right)$ is the distance from $x_{0}^{(j)}$ to the closest edge of the bottom boundary. The right column of Fig. 5 shows the area defined as the union of the balls with maximum radii,

$$
Q_{\max }:=\bigcup_{j=1}^{J}\left(B\left(x_{0}^{(j)}, R_{\max }\left(x_{0}^{(j)}\right)\right) \cap \Omega\right),
$$

i.e. $Q_{\max }$ is the area where the probing is performed.

Let $R_{\text {rec }}\left(x_{0}\right)$ be the approximation of the distance from $x_{0}$ to the inclusions obtained by the algorithm given in section 1 . If the largest ball does not intersect with any of the inclusions, we employ the maximum radius $R_{\max }\left(x_{0}\right)$ as $R_{\text {rec }}\left(x_{0}\right)$. Then the union of the balls with estimated radii, i.e.

$$
D_{\text {rec }}:=\bigcup_{j=1}^{J}\left(B\left(x_{0}^{(j)}, R_{\text {rec }}\left(x_{0}^{(j)}\right)\right) \cap \Omega\right)
$$

gives the area in which any inclusions do not exist.

\subsection{Enhancements of inclusion detection}

In the present study we incorporate three additional processes to enhance the reconstruction algorithm.

\subsubsection{Dirichlet data with rotation}

The detection algorithm introduced in section 1 inheres the problem of asymmetry of the Dirichlet data as discussed in subsection 2.2. Namely, the performance of the inclusion detection varies depending on the coordinate system due to the asymmetric transform in (2.2) even if the same probing ball is used. In order to address the problem, we use multiple Dirichlet data per one ball defined as rotations with different angles.

Let us assume a coordinate system $\left(x_{1}^{\prime}, x_{2}^{\prime}, x_{3}^{\prime}\right)$ originated at a given center point $x_{0}$ with a set of orthogonal bases, $\left\{e_{1}^{\prime}, e_{2}^{\prime}, e_{3}^{\prime}\right\}$, chosen so as to satisfy

$$
\Omega \subset\left\{x=x_{1}^{\prime} e_{1}^{\prime}+x_{2}^{\prime} e_{2}^{\prime}+x_{3}^{\prime} e_{3}^{\prime} \mid x_{3}^{\prime}>0\right\} .
$$

We define the rotation of the Dirichlet data with an angle $\theta$ as:

$$
f_{\tau}(x ; \theta)=\left.u\left(x_{1}^{\prime \prime}, x_{2}^{\prime \prime}, x_{3}^{\prime}\right)\right|_{\Gamma},
$$

where $\left(x_{1}^{\prime \prime}, x_{2}^{\prime \prime}\right)$ are the rotated coordinate of $\left(x_{1}^{\prime}, x_{2}^{\prime}\right)$ about $x_{3}^{\prime}$ axis with angle $\theta$. Fig. 4 shows examples of the Dirichlet data corresponding to angles $\theta=0, \pi / 2, \pi$ and $3 \pi / 2$, where the probing ball is taken at $x_{0}=(0.2,0.4,-0.2)$ and $R=0.5$. 
We define the reconstruction obtained using a rotation $\theta$ by

$$
D_{\text {rec }}(\theta):=\bigcup_{j=1}^{J}\left(B\left(x_{0}^{(j)}, R_{\text {rec }}\left(x_{0}^{(j)}, \theta\right)\right) \cap \Omega\right),
$$

where $R_{\text {rec }}\left(x_{0}^{(j)}, \theta\right)$ is the approximate distance from $x_{0}^{(j)}$ to the inclusion obtained using the rotated Dirichlet data with angle $\theta$. For the enhancement, we give multiple angles $\theta_{k}(k=1, \ldots, K)$ and obtain approximate distances, $n R_{\text {rec }}\left(x_{0}^{(j)}, \theta_{k}\right)$, for each center point. Then we define the reconstruction as the union of the balls with the approximated distances by

$$
Q_{\text {rec }}:=\bigcup_{k=1}^{K} D_{\text {rec }}\left(\theta_{k}\right)
$$

\subsubsection{Restriction of the maximum radius of probing balls}

The numerical tests shown in subsection 2.5 indicates that the inclusion detection algorithm is robust to noise if the distance to the inclusions is relatively small. However, as shown in the results in the right columns of Fig. 3, the value of the inner product is sensitive to the noise if the radius is relatively large and the noise causes large error in the estimated distance.

In the numerical study of this section, we restrict the depth of the probing in order to avoid the situation in which the noise results in large error in the estimated distance. Let $R_{\text {ceil }}$ be the maximum radius of probing balls given as a user defined parameter. We use $0.2<R<\max \left\{R_{\max }\left(x_{0}^{(j)}\right), R_{\text {ceil }}\right\}$ as the range of the probing balls.

\subsubsection{Stabilization of distance estimation process}

We assume that the Neumann data measured on the localized boundary contain noise $c \eta$ in the same way as in subsection 2.5. The inner product can be divided into the ideal part and the error part as follows:

$$
\left\langle\left(\Lambda f_{\tau}+c \eta\right)-\Lambda_{0} f_{\tau}, f_{\tau}\right\rangle_{\Gamma}=\left\langle\left(\Lambda-\Lambda_{0}\right) f_{\tau}, f_{\tau}\right\rangle_{\Gamma}+c\left\langle\eta, f_{\tau}\right\rangle_{\Gamma}
$$

This equation implies that the error part, $c\left\langle\eta, f_{\tau}\right\rangle_{\Gamma}$, can cause a fatal error in the inner product, $\left\langle\left(\Lambda f_{\tau}+c \eta\right)-\Lambda_{0} f_{\tau}, f_{\tau}\right\rangle_{\Gamma}$, if the absolute value of the ideal part, $\left|\left\langle\left(\Lambda-\Lambda_{0}\right) f_{\tau}, f_{\tau}\right\rangle_{\Gamma}\right|$, is smaller than or approximately equal to that of the error part, $\left|c\left\langle\eta, f_{\tau}\right\rangle_{\Gamma}\right|$. In such cases, the error affected by the noise in the measurements accounts for the majority of the computed inner product and hence the approximate distance obtained by the inclusion detection algorithm is not reliable.

Note that such a situation is the case when the ball does not intersect with the inclusion, i.e. the inclusion detection algorithm is sensitive to noise if the distance to the inclusion is larger than the radius of the ball. From this property, if the error part dominates the computed inner product, the ball can be estimated to have no intersection with the inclusion and the situation in which error dominates the computed inner product can be avoided.

We incorporate the idea described above as the process prior to the inclusion detection algorithm to avoid the use of the unreliable inner product. Assuming that 
the level of the noise part, $\left|c\left\langle\eta, f_{\tau}\right\rangle_{\Gamma}\right|$, is given, we set a threshold, $\varepsilon_{\mathrm{T}}>0$, depending on the level as follows:

$$
\varepsilon_{\mathrm{T}}=\beta\left|c\left\langle\eta, f_{\tau}\right\rangle_{\Gamma}\right|,
$$

where $\beta>0$ is a user defined parameter. If the computed inner product, $\left\langle\left(\Lambda f_{\tau}+\right.\right.$ $\left.c \eta)-\Lambda_{0} f_{\tau}\right\rangle_{\Gamma}$ is smaller than the threshold, we regard that the major part of the inner product is error and determine that the ball does not contain any inclusions. This process works as a stabilizer in the sense the infection of the measurement noise can be eliminated. Note that the level of the noise part can be estimated from multiple measurements if the noise distributes according to a certain probability model.

\subsection{Inclusions: numerical studies 1 (Recovery of inclusions)}

In the present numerical studies we assume two different inclusions: a square and a sphere as shown in Fig. 6 (a) and 7 (a), where the square is in $-0.5<x_{1}<$ $-0.2,-0.9<x_{2}<0,0.1<x_{3}<1.9$ and the sphere is centered at $(-0.6,-0.6,0.4)$ with radius 0.3 , respectively. Each of the figures also illustrates the goal of the recovery, i.e. the area that can be determined to be background if the accurate distances from all the center points to the noise are obtained. Here we set the maximum radius of the probing balls by $R_{\text {ceil }}=0.6$. We denote the area by $Q_{\text {best }}$.

Fig. 6 (b) and 7 (b) illustrate the recovered areas, $Q_{\text {rec }}$ in (3.2), obtained as results of numerical simulations using the inclusion detection algorithm. Here, the inner product, $\left\langle\left(\Lambda-\Lambda_{0}\right) f_{\tau}, f_{\tau}\right\rangle_{\Gamma}$, is computed using the Dirichlet data given in (3.1) with rotations $\theta=0, \pi / 2, \pi, 3 \pi / 2$ and corresponding Neumann data obtained by finite element method. The Neumann data in this numerical test does not contain noise except for the error of finite element approximation. The stabilizer in 3.2.3 is not used in this test since the Neumann data is not noisy. The relative error of the recovery for each case is also shown on the figure, where the relative error is defined as following:

$$
\frac{\mu\left(Q_{\text {best }} \backslash Q_{\text {rec }}\right)+\mu\left(Q_{\text {rec }} \backslash Q_{\text {best }}\right)}{\mu\left(Q_{\text {best }}\right)} \cdot 100 \%
$$

and $\mu$ stands for the volume. These results show that the area can be recovered by the inclusion detection algorithm within $5 \%$ error.

Fig. 6 (c-d) and 7 (c-d) are the results of the recovery using Neumann data with noise, $\Lambda f_{\tau}+c \eta$. The noise level, $c$, used in these figures are $1.0 \times 10^{-4}$ (see Fig. 6 (c) and 7 (c)) and $1.0 \times 10^{-3}$ (see Fig. 6 (d) and 7 (d)). The stabilizer given in 3.2.3 is not used in these computations. These results illustrate that, although the recovery is accurate around the inclusion, the area far from the inclusion contains large error. The error is caused by the inaccurate computation of the inner product as discussed in 3.2.3. The relative errors of the recoveries are $39.8 \%$ and $62.4 \%$ in the case of $c=1.0 \times 10^{-4}$ and are $63.7 \%$ and $80.2 \%$ in the case of $c=1.0 \times 10^{-3}$.

Fig. $6(\mathrm{e}-\mathrm{f})$ and $7(\mathrm{e}-\mathrm{f})$ are the results of the reconstruction obtained with the stabilizer proposed in 3.2.3. Here, we set $\varepsilon_{\mathrm{T}}=5.0 \times 10^{-4}$ as the threshold for the stabilization in (3.3). These figures illustrate that the influence of the inaccurate computations caused by the measurement noise is effectively removed by the stabilizer and the areas that are far from the inclusion can be accurately recovered.

\subsection{Inclusions: numerical studies 2 (Distance to spherical inclusion)}

The purpose of this numerical test is to verify the effectiveness of our algorithm by comparing with the method of small inhomogeneity detection proposed in [12]. We 
Table 1. Inclusion detection: estimated center of spherical inclusion

\begin{tabular}{|l|l|l|l|l|l|l|}
\hline & \multicolumn{2}{|c|}{$0 \%$} & \multicolumn{2}{c|}{$0.5 \%$} & \multicolumn{2}{c|}{$1.0 \%$} \\
\hline $\begin{array}{l}\text { z-coord. } \\
\text { of center }\end{array}$ & $\begin{array}{l}\text { Estimated } \\
\text { z-coord. }\end{array}$ & $\begin{array}{l}\text { Relative } \\
\text { error }\end{array}$ & $\begin{array}{l}\text { Estimated } \\
\text { z-coord. }\end{array}$ & $\begin{array}{l}\text { Relative } \\
\text { error }\end{array}$ & $\begin{array}{l}\text { Estimated } \\
\text { z-coord. }\end{array}$ & $\begin{array}{l}\text { Relative } \\
\text { error }\end{array}$ \\
\hline $0.15(\mathrm{D} 1)$ & 0.144 & $3.8 \%$ & 0.142 & $5.5 \%$ & 0.139 & $7.3 \%$ \\
\hline $0.20(\mathrm{D} 2)$ & 0.189 & $5.4 \%$ & 0.172 & $14.1 \%$ & 0.166 & $16.8 \%$ \\
\hline $0.25(\mathrm{D} 3)$ & 0.226 & $9.6 \%$ & 0.197 & $21.0 \%$ & 0.194 & $22.6 \%$ \\
\hline
\end{tabular}

take the same cubic region as in section 2 and consider a problem to approximate the center of an inclusion in order to compare the results with those described in [12]. We assume three different spherical inclusions, D1, D2 and D3, with radius 0.1, where the centers are at $(0,0,0.15),(0,0,0.2)$ and $(0,0,0.25)$, respectively. We also assume that the radius of each inclusion is given and that the conductivity of the background and the inclusions 1 and $2 / 3$, respectively. Note that the geometry is the same as the numerical tests in [12] except for its scale and complexity of the background conductivity. We calculate an approximate z-coordinate of the center of the spherical inclusion using the distance from $x_{0}$ to the inclusion, where $x_{0}=(0,0,-0.2)$.

Tab. 1 shows the z-coordinates of the estimated centers of the spherical inclusions together with their relative errors for different noise levels, $0 \%, 0.5 \%$ and $1.0 \%$. Although the results can not be simply compared to those in [12], our results indicate that the relative errors are almost the same or smaller than in [12] and that reliable results can be obtained especially when the distance from the boundary to inclusions is small even if the measurements contain noise.

\section{Conclusion}

We have shown a method of three-dimensional electrical impedance tomography for finding electrical inclusions in an object. The detection algorithm works using electrical measurements performed on a localized boundary that enables partial recovery of the shape of inclusions.

Our numerical experiments with simulated noisy data suggest that the method can be used for extracting information about inclusions (located relatively close to the boundary) in a reliable and robust way. Moreover, the Dirichlet data used in the method can be approximated by a practical number of electrodes.

In addition to the requirement that the inclusions should be relatively close to the boundary, it is also important that the inclusions are not too small. Namely, according to the distinguishability analysis by David Isaacson [52], with a given noise amplitude any inclusion smaller than a limit size does not contribute enough to the measurements to be detectable.

Potential applications of the method include early diagnosis of breast cancer, underground contaminant detection and nondestructive testing.

\section{Acknowledgements}

During part of the preparation of this work, SS worked as professor at the Department of Mathematics of Tampere University of Technology. Also, the work of SS was funded by the Finnish Centre of Excellence in Inverse Problems Research 
(Academy of Finland CoE-project 213476).

\section{References}

[1] Alessandrini G 1988 Stable determination of conductivity by boundary measurements Appl. Anal. 27 153-172

[2] Alessandrini G 1990 Singular solutions of elliptic equations and the determination of conductivity by boundary measurements J. Diff. Eqns $84252-272$

[3] Alessandrini G, Bilotta A, Morassi A, Rosset E and Turco E 2007 Computing volume bounds of inclusions by EIT measurements J Sci Comput 33, 293-312

[4] Alessandrini G and Di Cristo M 2005 Stable determination of an inclusion by boundary measurements SIAM J. Math. Anal. 37 200-217

[5] Alessandrini G and Isakov V 1996 Analyticity and uniqueness for the inverse conductivity problem Rend. Istit. Mat. Univ. Trieste 28 351-369

[6] Alessandrini G, Morassi A and Rosset E 2003 Size estimates Contemporary Mathematics 333

[7] Alessandrini G and Rosset E 1998 The inverse conductivity problem with one measurement: bounds on the size of the unknown object SIAM J. Appl. Math. 58 1060-1071

[8] Alessandrini G and Rosset E 2004 Volume bounds of inclusions from physical EIT measurements Inverse Problems 20 575-588

[9] Alessandrini G, Rosset E, Seo J K 2000 Optimal size estimates for the inverse conductivity problem with one measurement Proc. Amer. Math. Soc. 128 53-64

[10] Ammari H, An Introduction to Mathematics of Emerging Biomedical Imaging, Math. \& Appl., Vol. 62, Springer-Verlag, Berlin, 2008.

[11] Ammari H, Griesmaier R and Hanke M 2007 Identification of small inhomogeneities: asymptotic factorization Math. Comp. 76 1425-1448

[12] Ammari H and Kang H 2004, Reconstruction of Small Inhomogeneities from Boundary Measurements, Lecture Notes in Mathematics, Vol. 1846, Springer-Verlag, Berlin, 2004.

[13] Ammari H and Kang H 2006 Generalized polarization tensors, inverse conductivity problems, and dilute composite materials: a review. Contemp. Math. 408 1-67

[14] Ammari $\mathrm{H}$ and Kang H, Polarization and Moment Tensors: with Applications to Inverse Problems and Effective Medium Theory, Applied Mathematical Sciences, Vol. 162, SpringerVerlag, New York, 2007.

[15] Ammari H, Kang H, Kim E and Lim M 2005 Reconstruction of closely spaced small inclusions SIAM J. Numer. Anal. 42 2408-2428

[16] Ammari H, Kwon O, Seo J K, and Woo E J 2004 Anomaly detection in T-scan trans-admittance imaging system, SIAM J. Appl. Math., 65 (2004), 252-266.

[17] Barcelò B, Fabes E and Seo K 1994 The inverse conductivity problem with one measurement: uniqueness for convex polyhedra Proc. Amer. Math. Soc. 122 183-189

[18] Bikowski J 2008 Electrical Impedance Tomography reconstructions in two and three dimensions; From Calderón to Direct Methods, PhD Thesis, Colorado State University

[19] Blue R 1997 Real-time three-dimensional electrical impedance tomography, PhD thesis, Rensselaer Polytechninc Institute, Troy, New York

[20] Boverman G, Isaacson D, Kao T-J, Saulnier G J and Newell J C 2008 Methods for Direct Image Reconstruction for EIT in Two and Three Dimensions Proceedings of the 2008 Electrical Impedance Tomography Conference took place at Dartmouth College, in Hanover, New Hampshire, USA, June 16 to 18, 2008

[21] Brown R M 1996 Global uniqueness in the impedance-imaging problem for less regular conductivities SIAM J. Math. Anal. 27 1049-1056

[22] Brühl M 2001 Explicit characterization of inclusions in electrical impedance tomography SIAM J. Math. Anal. 32 1327-1341

[23] Brühl M and Hanke M 2000 Numerical implementation of two non-iterative methods for locating inclusions by impedance tomography, Inverse Problems 161029

[24] Bukhgeim A L and Uhlmann G 2002 Recovering a potential from partial Cauchy data Comm. $P D E 27$ 653-668

[25] Calderón A P 1980 On an inverse boundary value problem Seminar on numerical analysis and its applications to continuum physics (Soc. Brasileira de Matemàtica 1980), 65-73.

[26] Capdeboscq Y and Vogelius M S 2004 A review of some recent work on impedance imaging for inhomogeneities of low volume fraction. Contemp. Math. 362 69-87 
[27] Chanillo S 1990 A problem in electrical prospection and an n-dimensional Borg-Levinson theorem Proc. Amer. Math. Soc. 108 761-767

[28] Cheney M, Isaacson D and Newell J C 1999 Electrical Impedance Tomography SIAM Review 41 85-101

[29] Cook R D, Saulnier G J and Goble J C 1991 A phase sensitive voltmeter for a high-speed, high precision electrical impedance tomograph. Annual International Conference of the IEEE Engineering in Medicine and Biology Society 13, pp. 22-23

[30] Cornean H, Knudsen K and Siltanen S 2006 Towards a d-bar reconstruction method for threedimensional EIT. Journal of Inverse and Ill-Posed Problems 14 111-134

[31] Daido Y and Nakamura G 2004 Reconstruction of inclusions for the inverse boundary value problem with mixed type boundary condition and source term Inverse Problems 201599 1619

[32] Erhard K and Potthast R 2003 The point source method for reconstructing an inclusion from boundary measurements in electrical impedance tomography and acoustic scattering Inverse Problems 19 1139-1157

[33] Friedman A 1987 Detection of mines by electric measurements SIAM J. Appl. Math. 47 201-212

[34] Friedman A and Gustafsson B 1987 Identification of the conductivity coefficient in an elliptic equation SIAM J. Math. Anal. 18 777-787

[35] Friedman A and Isakov V 1989 On the uniqueness in the inverse conductivity problem with one measurement Indiana Univ. Math. J. 38 563-579

[36] Gebauer B and Hyvönen N 2007 Factorization method and irregular inclusions in electrical impedance tomography Inverse Problems 23 2159-2170

37] Gebauer B and Hyvönen N 2008 Factorization method and inclusions of mixed type in an inverse elliptic boundary value problem Inverse Probl. Imaging 2 355-372

[38] Goble J, Cheney M and Isaacson D 1992 Electrical impedance tomography in three dimensions Appl Comput Electromagn Soc J 7 128-147

[39] Greenleaf A, Lassas M and Uhlmann G 2003 The Calderón Problem for Conormal Potentials I: Global Uniqueness and Reconstruction Comm. Pure Appl. Math 56 328-352

[40] Hanke M and Schappel B 2008 The Factorization Method for Electrical Impedance Tomography in the Half-Space SIAM J. Appl. Math. 68907 骞-924

[41] Peter Hähner 1999 An inverse problem in electrostatics Inverse Problems 15 961-975

[42] Hyvönen N 2007 Application of the factorization method to the characterization of weak inclusions in electrical impedance tomography Adv. in Appl. Math. 39 197-221

[43] Ide T, Isozaki H, Nakata S, Siltanen S and Uhlmann G 2007 Probing for electrical inclusions with complex spherical waves Communications on Pure and Applied Mathematics 60 1415-1442

[44] Ikehata M 1998 Reconstruction of the shape of the inclusion by boundary measurements, Comm PDE 23 1459-1474

[45] Ikehata M 1998 Size estimation of inclusion J. Inverse Ill-Posed Probl. 6 127-140

[46] Ikehata M 2000 Reconstruction of the support function for inclusion from boundary measurements J. Inverse Ill-Posed Probl. 8 367-378

[47] Ikehata M 2001 Inverse conductivity problem in the infinite slab Inverse Problems 17 437-454

[48] Ikehata M 2003 Extracting the Convex Hull of an Unknown Inclusion in the Multilayered Material Applicable Analysis 82 857-873

[49] Ikehata M and Nakamura G 1999 Slicing of a three-dimensional object from boundary measurements Inverse Problems 15 1243-1253

[50] Ikehata M and Siltanen S 2000 Numerical method for finding the convex hull of an inclusion in conductivity from boundary measurements Inverse Problems 16 1043-1052

[51] Ikehata M and Siltanen S 2004 Electrical impedance tomography and Mittag-Leffler's function, Inverse Problems 20 1325-1348

[52] Isaacson D 1986 Distinguishability of Conductivities by Electric Current Computed Tomography, IEEE Tr. Medical Imaging, Vol 5, pp. 91-95.

[53] Isakov V 1988 On uniqueness of recovery of a discontinuous conductivity coefficient Commun. Pure. Appl. Math. 41 865-877

[54] Isakov V 2007 On uniqueness in the inverse conductivity problem with local data Inverse Probl. Imaging 1 95-105

[55] Kang H, Seo J K and Sheen D 1997 Numerical identification of discontinuous conductivity coefficients Inverse Problems 13 113-123

[56] Kenig C E, Sjöstrand J and Uhlmann G 2007 The Calderón problem with partial data Ann. of Math. 165 567-591

[57] Kim S 2006 An inverse boundary value problem of determining three dimensional unknown inclusions in an elliptic equation J. Inverse Ill-Posed Probl. 14 881-889 
[58] Knudsen K 2006, The Calderón problem with partial data for less smooth conductivities Comm. Partial Differential Equations 31 57-71.

[59] Kohn R V and Vogelius M 1985 Determining conductivity by boundary measurements II. Interior results. Commun. Pure Appl. Math. 38 643-667

[60] Kwon O and Seo J K 2001, Total size estimation and identification of multiple anomalies in the inverse conductivity problem, Inverse Problems 17 59-75

[61] Kwon O, Seo J K, and Yoon J R 2002 A real-time algorithm for the location search of discontinuous conductivities with one measurement, Comm. Pure Appl. Math., 55 (2002), $1-29$.

[62] Lechleiter A, Hyvönen N and Hakula H 2008 The factorization method applied to the complete electrode model of impedance tomography SIAM J Appl Math 68 1097-1121

[63] Lesnic D 2002 Inversion of discontinuous anisotropic conductivities, Recent Development in Theories 85 Numerics: International Conference on Inverse Problems, Hong Kong, China, 9-12 January 2002, Yiu-Chung Hon (Ed.), World Scientific, 2002

[64] Metherall P, Barber D C, and Smallwood R M 1995 Three dimensional electrical impedance tomography In Proc IX Int Conf Electrical Bio-Impedance, 510-511, Heidelberg, Germany

[65] Metherall P, Barber D C, Smallwood R H and Brown B H 1996 Three-dimensional electrical impedance tomography Nature $\mathbf{3 8 0} 509-512$

[66] Metherall P, Smallwood R H, and Barber D C 1996 Three dimensional electrical impedance tomography of the human thorax In Proc 18th Int Conf IEEE Eng Med Biol Society

[67] Morucci J P, Granie M, Lei M, Chabert M, and Marsili P M 1995 3D reconstruction in electrical impedance imaging using a direct sensitivity matrix approach Physiol Meas 16 A123-A128

[68] Mueller J L, Isaacson D and Newell J 1999 A Reconstruction Algorithm for Electrical Impedance Tomography Data Collected on Rectangular Electrode Arrays IEEE Trans. Biomed. Engr. 46 1379-1386

[69] Mueller J L, Isaacson D and Newell J 2001 Reconstruction of Conductivity Changes Due to Ventilation and Perfusion from EIT Data Collected on a Rectangular Electrode Array Physiol. Meas. 22 97-106

[70] Nachman A I 1988 Reconstructions from boundary measurements Ann. of Math. 128 531-576

[71] Nachman A I and Street B 2009 Reconstruction in the Calderón Problem with Partial Data arXiv:0904.4794

[72] Nachman A I, Sylvester J and Uhlmann G 1988 An n-dimensional Borg-Levinson theorem Communications in Mathematical Physics 115 595-605

[73] Novikov R G 1988 A multidimensional inverse spectral problem for the equation $-\Delta+(v(x)-$ $E u(x))=0$ Funct. Anal. Appl. 22 263-272

[74] Päivärinta L, Panchenko A and Uhlmann G 2003 Complex geometrical optics solutions for Lipschitz conductivities Rev. Mat. Iberoamericana 19 56-72

[75] Sylvester J and Uhlmann G 1987 A global uniqueness theorem for an inverse boundary value problem Ann. of Math. 125 153-169

[76] Uhlmann G and Wang J-N 2008 Reconstructing Discontinuities Using Complex Geometrical Optics Solutions SIAM J. Appl. Math. 68 1026-1044

[77] Vauhkonen P J 2004 Image Reconstruction in Three-Dimensional Electrical Impedance Tomography Phd thesis, Kuopio University publications C166

[78] Vauhkonen P J, Vauhkonen M, Savolainen T, and Kaipio J P 1999 Static three-dimensional electrical impedance tomography Ann New York Acad Sci $\mathbf{8 7 3} 472-481$

[79] Vauhkonen P J, Vauhkonen M, Savolainen T, and Kaipio J P 1999 Three-dimensional electrical impedance tomography based on the complete electrode model. IEEE Trans. Biomed. Eng. 46 1150-1160

[80] Wexler A 1988 Electrical impedance imaging in two and three dimensions. Clin Phys Physiol Meas, Suppl A 9 29-33 
Local detection of three-dimensional inclusions in electrical impedance tomography 14

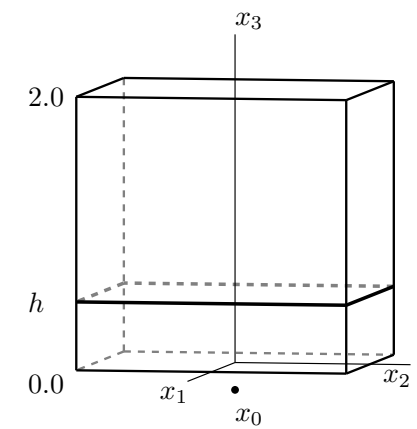

Figure 1. Geometry of test problems with inclusion. $h:$ height of the interface, $x_{0}=(-0.2,0,0):$ center of probing balls 
Local detection of three-dimensional inclusions in electrical impedance tomography 15
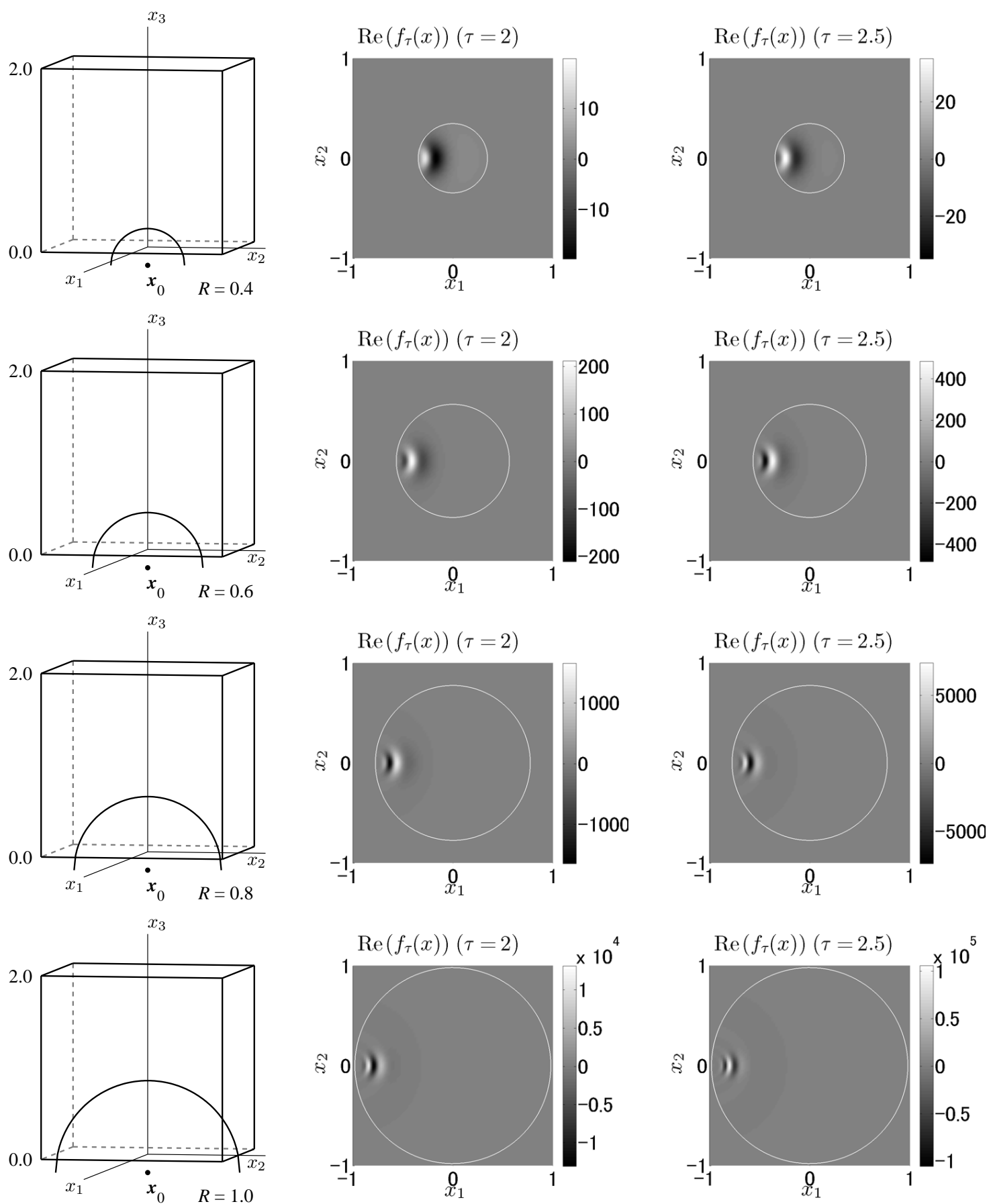

Figure 2. Real part of Dirichlet data given at the bottom boundary. Left: Geometry of test problems with probing sphere. Center: Real part of boundary condition with $\tau=2.0$, Right: Real part of boundary condition with $\tau=2.5$. White lines denote the boundary of the localized area in which the measurements are performed. 
Local detection of three-dimensional inclusions in electrical impedance tomography 16
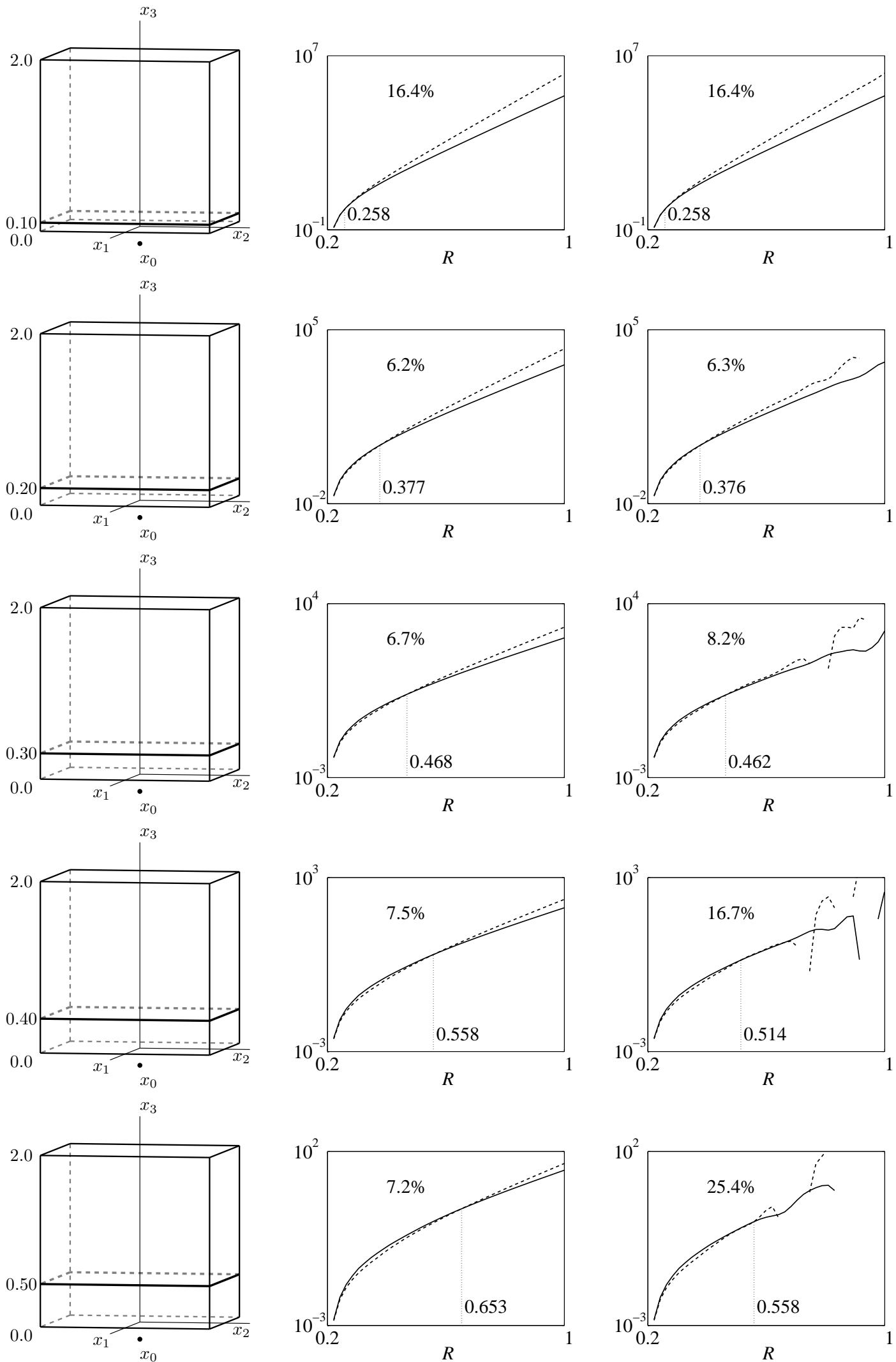

Figure 3. Left column: Geometry of layerd medium test problems. The problem domain is $[-1,1] \times[-1,1] \times[0,2]$ and the inclusion is $[-1,1] \times[-1,1] \times[h, 2]$, where $h=0.2,0.4,0.6$ and 0.8 , respectively. The center of spheres used for probing, $x_{0}$, is fixed to $(0,0,-0.2)$ throughout this test. Solid line: $I_{1}\left(\tau_{1}=2.0\right)$, dashed line: $I_{2}\left(\tau_{2}=2.5\right)$. 
Local detection of three-dimensional inclusions in electrical impedance tomography 17
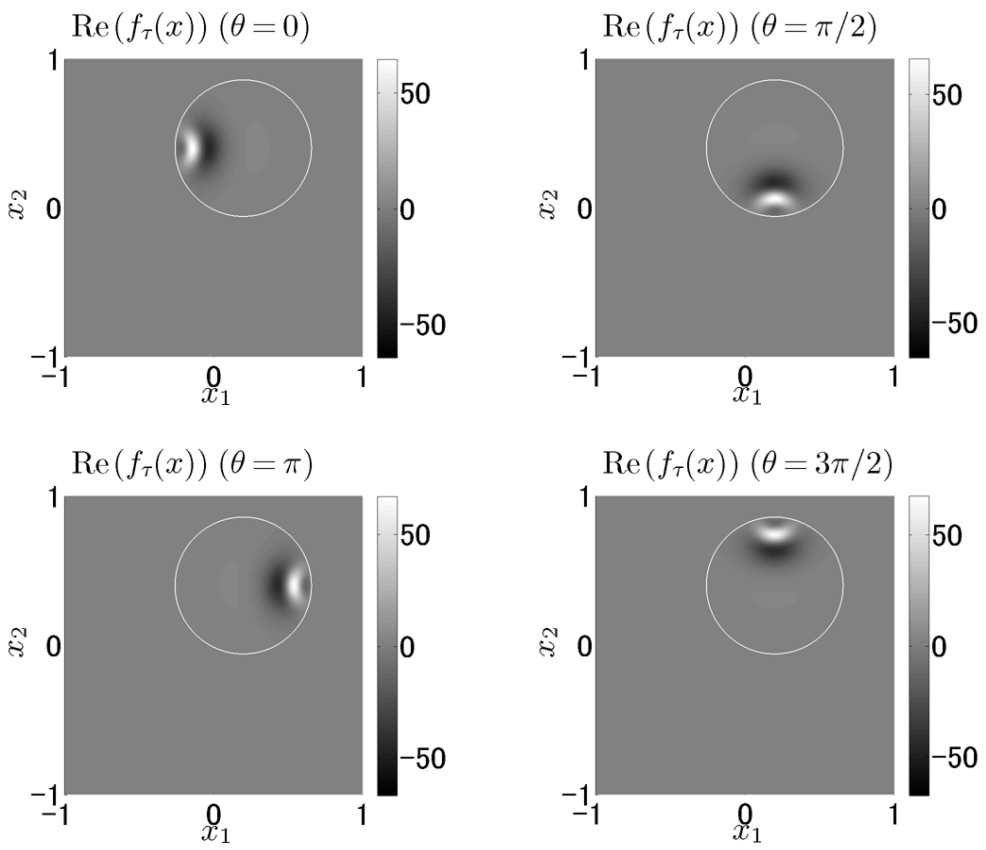

Figure 4. Examples of Dirichlet data with rotation for $\theta=0, \pi / 2, \pi, 3 \pi / 2$, where $x_{0}=(0.2,0.4,-0.2)$ and $R=0.5$.
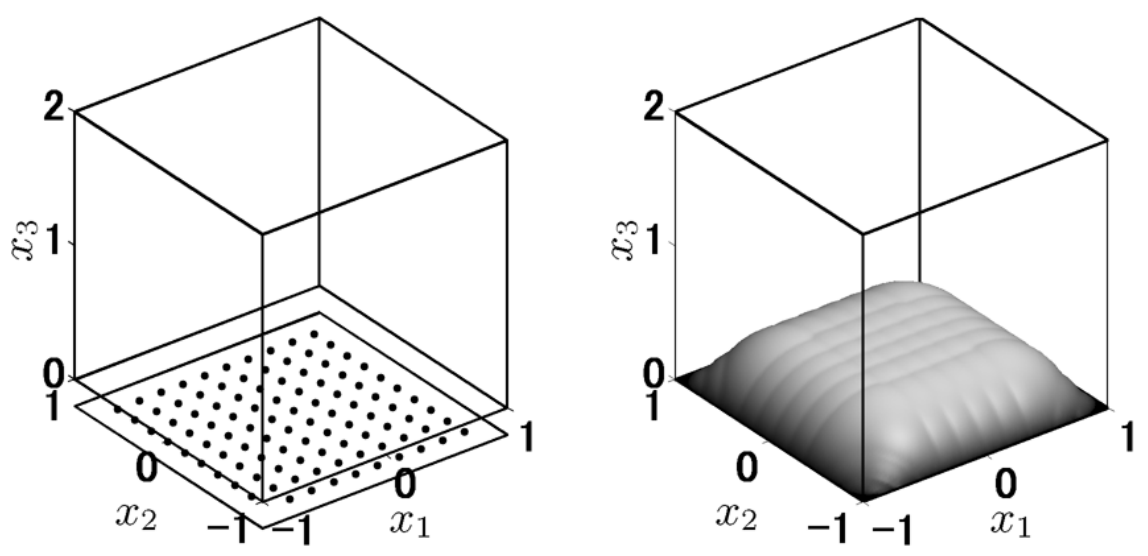

Figure 5. Geometry of inclusion detection problem. Left: Center points of probing spheres $\left(x_{3}=-0.2\right)$. Right: Probing region that probing spheres reach from the center points. 


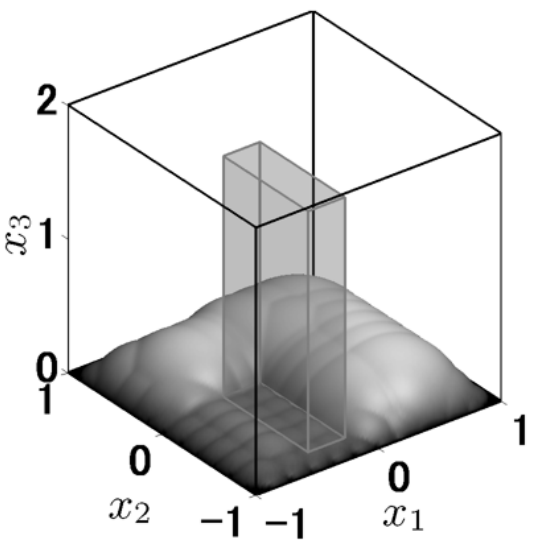

(a) ideal recovery $Q_{\text {best }}$

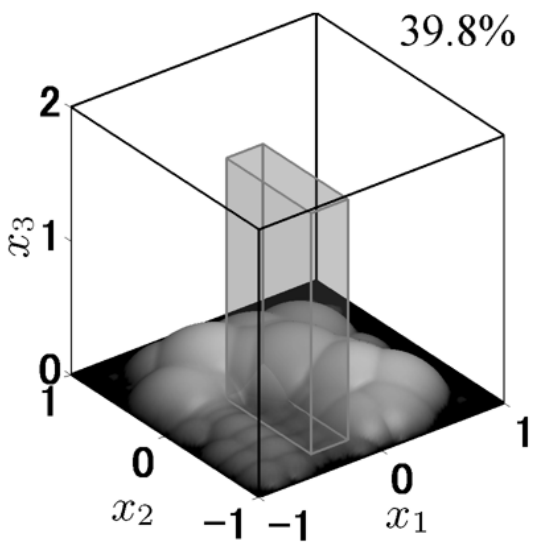

(c) $Q_{\text {rec }}$ with $0.01 \%$ noise

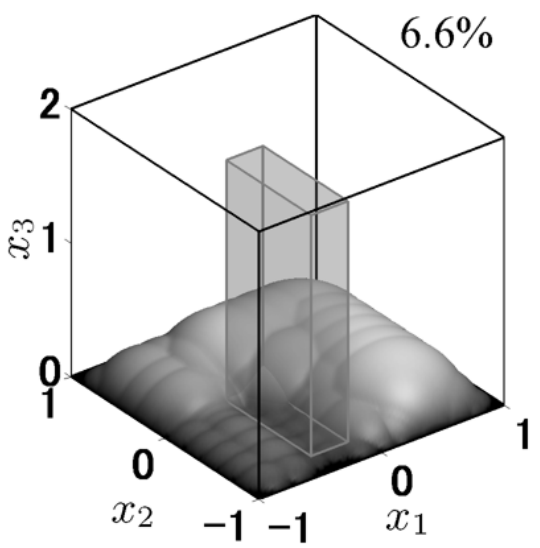

(e) $Q_{\text {rec }}$ with $0.01 \%$ noise (stabilized)

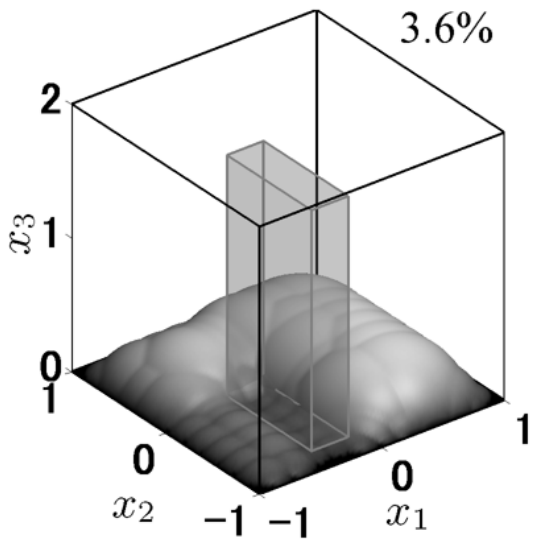

(b) $Q_{\text {rec }}$ without noise

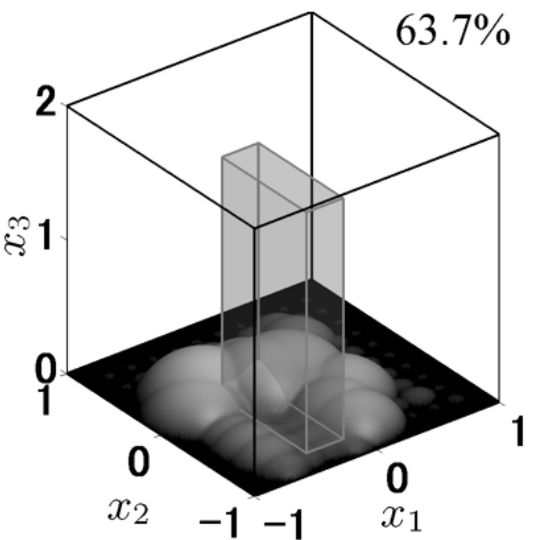

(d) $Q_{\text {rec }}$ with $0.1 \%$ noise

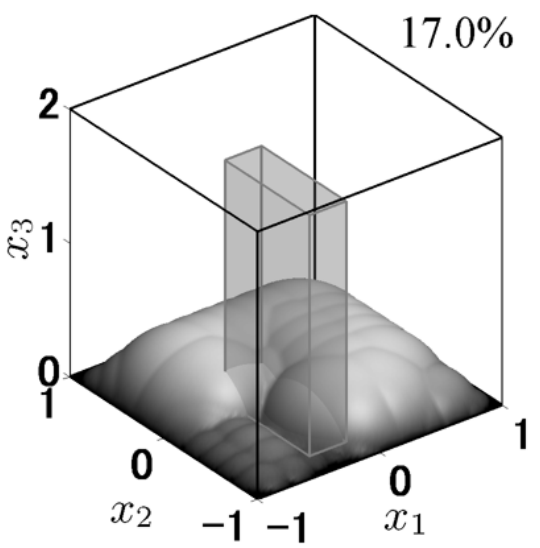

(f) $Q_{\text {rec }}$ with $0.1 \%$ noise (stabilized)

Figure 6. Results of inclusion detection (a square inclusion). The percenteges shown are relative errors of reconstructions as defined in formula (3.4). Compare the middle row and the bottom row to see how our stabilization approach improves the reconstruction significantly when the measurement data contains noise of realistic amplitude. 


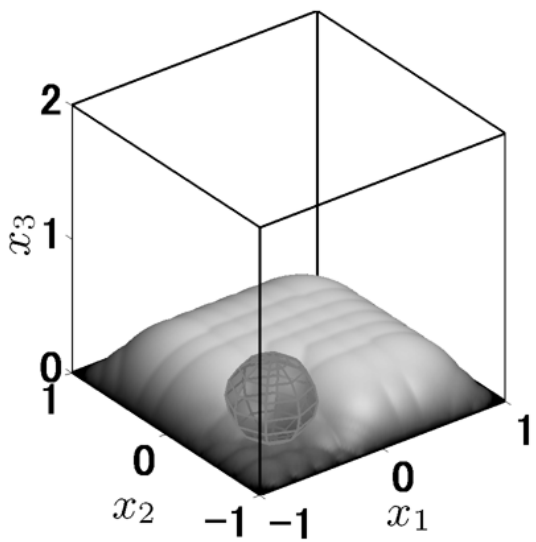

(a) ideal recovery $Q_{\text {best }}$

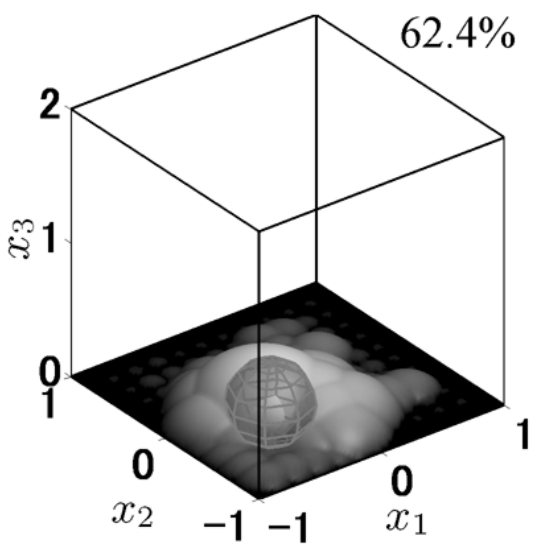

(c) $Q_{\text {rec }}$ with $0.01 \%$ noise

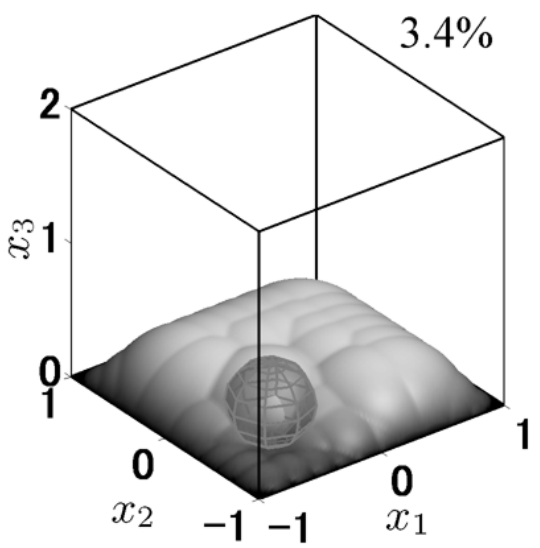

(e) $Q_{\text {rec }}$ with $0.01 \%$ noise (stabilized)

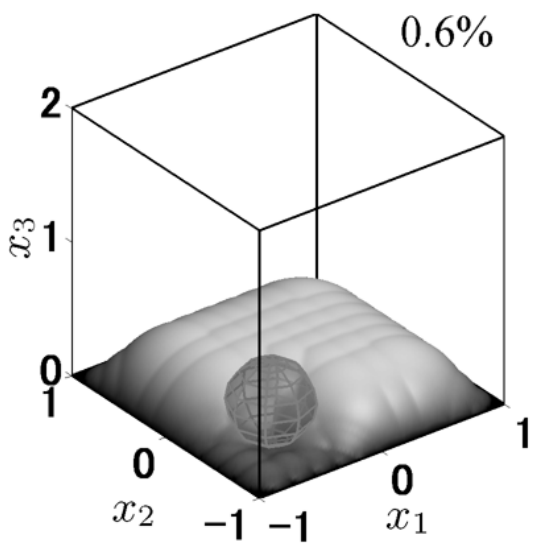

(b) $Q_{\text {rec }}$ without noise

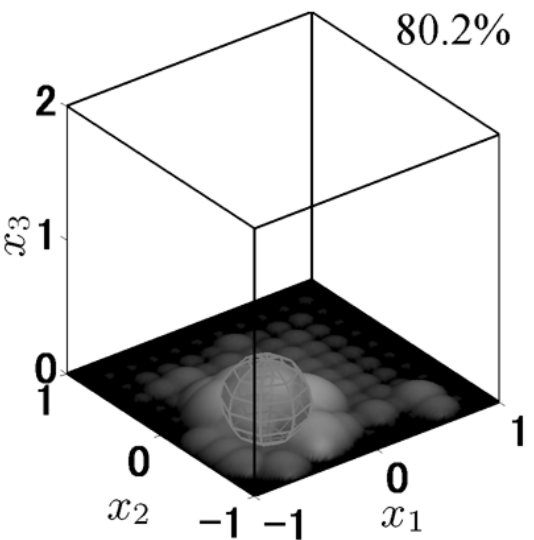

(d) $Q_{\text {rec }}$ with $0.1 \%$ noise

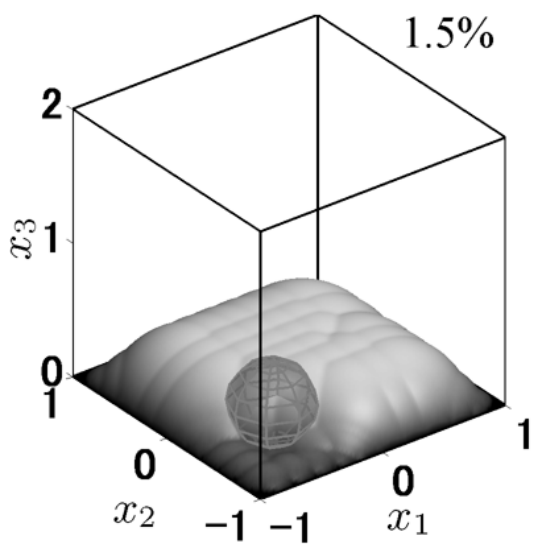

(f) $Q_{\text {rec }}$ with $0.1 \%$ noise (stabilized)

Figure 7. Results of inclusion detection (a spherical inclusion). The percenteges shown are relative errors of reconstructions as defined in formula (3.4). Compare the middle row and the bottom row to see how our stabilization approach improves the reconstruction significantly when the measurement data contains noise of realistic amplitude. 